\title{
Le projet AGUST
}

\section{Analyse des convergences et des divergences entre des procédés psychothérapeutiques spécifiques à des écoles}

\author{
Cornelia Stegmann, Rosmarie Barwinski, Katrin Hartmann, Mario Schlegel \& Agnes von Wyl
}

Psychotherapie-Wissenschaft 9 (2) 85-86 2019

www.psychotherapie-wissenschaft.info

CC BY-NC-ND

https://doi.org/10.30820/1664-9583-2019-2-85

Mots clés : recherche en psychothérapie, interventions psychothérapeutiques, facteurs efficaces, écoles de psychothérapie

\begin{abstract}
De quelle manière est-ce que la procédure pratique concrète appliquée dans les psychothérapies se différencie - ou non - en fonction des concepts théoriques sousjacents des différentes orientations théoriques des écoles ? Cette question a été explorée dans le projet AGUST qui s'est déroulé dans le cadre des colloques de recherche de l'ASP. AGUST est l'acronyme allemand : analyse des convergences et des divergences de procédés (psycho) thérapeutiques spécifiques à des écoles. Le projet a été initié et accompagné techniquement par la commission scientifique (WiKo) de l'ASP.
\end{abstract}

Entre septembre 2018 et janvier 2019, 24 psychothérapeutes ont analysé ensemble une vidéo de l'APA sur la toile de fond de différents contextes théoriques d'écoles. La vidéo montre la mise en œuvre par Norka Malberg de la thérapie basée sur la mentalisation d'après Fonagy au sein d'une séance présentant le cas d'une jeune femme confrontée à des difficultés de détachement décrites et souhaitant nouer une relation saine avec sa mère. Les participants répartis dans cinq groupes de travail (gestalt-psychothérapie, psychothérapie humaniste, psychanalyse de Jung, psychanalyse de Freud et psychothérapie orientée processus) ont, après l'évaluation des interventions dans la vidéo, été questionnés et priés de formuler des interventions psychothérapeutiques et des objectifs alternatifs et se sont vu demander de motiver la mise en œuvre de leurs interventions sur la base des fondamentaux théoriques de leur école.

L'objet fondamental de la réflexion analytique était constitué par l'intervention concrète au sens d'une réaction linguistique des thérapeutes. Deux séquences filmées de la vidéo ont été montrées à cet effet (colloque d'introduction et d'approfondissement), et la totalité du film a été visionnée au troisième temps de relevé (colloque consacré aux théories). Les composantes supposées du problème de la cliente et le déroulement du contre-transfert d'après la vidéo ont en outre fait l'objet d'un questionnement sur la totalité du film. Les participants ont ensuite été priés d'exposer les fondamentaux spécifiques de leur école d'après l'exemple de la cliente de la vidéo. Les participants ont traité les questions pendant les colloques et parfois entre les colloques. Ils ont, dans le cadre de présentations plénières, présenté leurs résultats qui ont été transcrits et ont pu être repris par écrit pour des compléments apportés par les groupes. Les indications ont été analysées au moyen d'une analyse qualitative de contenu et du manuel PAP-S-Rating. Ceci a permis de répartir les propositions d'intervention selon des facteurs efficaces généraux, spécifiques et spécifiques à des écoles et de comparer les concepts théoriques des écoles entre eux.

Les personnes interrogées ont identifié dans les deux séquences vidéo la méthode de psychothérapie basée sur la mentalisation appliquée dans les deux séquences vidéo APA et ont considéré que l'accent était mis sur la clarification (clarifier, questionner). Pour leurs propositions d'interventions alternatives ont été choisies des réactions thérapeutiques qui soutiennent le traitement émotionnel et cognitif de la cliente. Ce sont essentiellement des facteurs spécifiques et spécifiques à des écoles qui ont été choisis par les participants eux-mêmes. Les objectifs thérapeutiques alternatifs proposés permettent de reconnaître à la fois la perspective de clarification et la perspective de maîtrise des participants. Les interventions alternatives concrètes ont compris à la fois des interventions à la fois générales, spécifiques et spécifiques à des écoles, comme cela a pu être reconnu grâce à l'attribution PAP-S-R-M.

Les groupes de travail ont surtout justifié la mise en œuvre de leurs interventions psychothérapeutiques alternatives par l'intention de soutenir le traitement émotionnel de la cliente $(37 \%)$. En deuxième position venait le traitement cognitif $(26 \%)$, suivi des efforts visant à stimuler la capacité à surmonter le problème $(21 \%)$. Le renforcement de la relation et de la motivation $(16 \%)$ occupait la quatrième place des justifications des groupes par rapport au choix de leurs interventions alternatives.

Les composantes supposées du problème de la cliente comprenaient des problèmes de détachement, la peur et la solitude ainsi que des problèmes d'estime de soi au sens le plus large du terme. Les groupes de travail ont ici mis l'accent sur des points différents. Un résultat intéressant a pu être souligné à propos de l'illustration du contexte propre spécifique à l'école. Les participants ont été priés de formuler des interventions permettant de visualiser le 
concept théorique de leur école au moyen d'une vidéo APA. Les participants ont aussi indiqué des facteurs à la fois généraux, spécifiques et spécifiques à des écoles pour illustrer l'arrière-plan théorique spécifique à leur école. Ce résultat montre à nouveau que les psychothérapeutes recourent à des interventions transcendant plusieurs écoles pour pouvoir atteindre leurs objectifs thérapeutiques.

Le projet AGUST a permis de se faire une impression sur la répartition des facteurs efficaces sur différentes orientations théoriques d'école. Le PAP-S-Rating utilisé a pu être vérifié du point de vue de son actualité et être utilisé en tant qu'instrument adapté pour l'analyse vidéo. L'échange collégial qui a accompagné le projet AGUST au plan scientifique semble en outre parfaitement convenir pour discuter les convergences et les divergences entre différents concepts théoriques d'école, et pourrait continuer à être utilisé à l'avenir à cet effet.

\section{Les auteures et auteurs}

Cornelia Stegmann étudie à la ZHAW, département psychologie appliquée.

Le Prof. Dr. Rosmarie Barwinski, la MSc Katrin Hartmann, le Dr. Mario Schlegel et le Prof. Dr. Agnes von Wyl sont membres de la WiKo de l'ASP.

\section{Contact}

E-Mail : cornelia.stegmann@icloud.com 